\title{
Die Zeremonialprotokolle und weitere Quellen zum Zeremoniell des Kaiserhofes im Wiener Haus-, Hof- und Staatsarchiv
}

\author{
Von Mark Hengerer
}

\section{Einleitung}

Die Geschichtswissenschaft ist seit den späten 1970er Jahren der Aufforderung von Norbert Elias, die Zeremonien im Schlafzimmer König Ludwigs XIV. von Frankreich nicht als „Kuriosum" zu betrachten sondern „sie dermaßen Schritt für Schritt zum Leben zu bringen, daß es möglich wird, in ihnen Aufbau und Funktionsweise der höfischen Figuration, aus

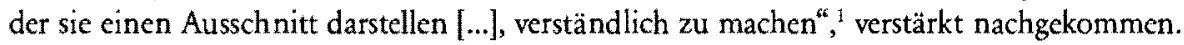
Zunächst hat sie dabei vorrangig die von Elias postulierte Instrumentalisierung des Hofzeremoniells als Herrschaftsinstrument kritisiert und damit einer ertragreichen Revision des klassischen Absolutismusparadigmas Vorschub geleistet. ${ }^{2}$ Besonders vor dem Hintergrund der von Literaturwissenschaftlern und Kunsthistorikern intensiv betriebenen Forschung zur frühneuzeitlichen Hofkritik und Konversationslehre sowie zum Hof als Hort repräsentativer Kunstformen boten sich im Rahmen dieser Revision mannigfaltige Möglichkeiten einer Einordnung des Zeremoniells in die jeweiligen Hofmodelle. Die von der Strukturgeschichte bis zur Semiotik reichende Palette der Ansätze wurde im Zuge des linguistic, des sociological, des cultural und jüngst des performative turn noch erheblich erweitert. ${ }^{3}$

So wird man heute die Anekdote, nach welcher Kaiser Karl VI. sich an der Schwelle des Todes darüber beschwert haben soll, daß am Fuße seines Bettes nur zwei statt der ihm als Kaiser gebührenden vier Kerzen standen, wohl nicht mehr in dem Sinne interpretieren, daß der "Kaiser in seinem Hofzeremoniell versponnen", also in irrationalen Verhaltensformen

I EuAs (1983) 126. -- Fur die freundliche Unterstutzung danke ich Thomas Just und Irmgard Pangerl vom HHStA.

2 Für den Diskussionswerlauf wurden KRUEDENER (1973) und EHAT (1980) mit der Systematisierung bzw. der Adaption für den Kaiserhof bedeutsam; darauf setzte mit WINTERLING (1986) die Revision der Funktionalisierung des Zeremoniells als Herrschaftsinstrument ein, die DUINDAM (1994 und 1998) im Rahmen einer breiteren Kritik [SCFW/ERHOF (1998)] vorantrieb. Wegmarken der Diskussion des Absolutismusparadigmas sind besonders HENSHAL (1992), ASCH/DUCHHARDT (1996) und ASCH (2001).

3 Bis in die 1990er Jahre waren vornehmlich literaturwissenschaftliche Arbeiten Wegmarken der interdisziplinär ausgerichteten Beschäftigung mit Zeremoniell, besonders KIESEL (1979), BUCK/KAUFFANN/SPAH/WEDEMANN (1981), FrCHSORGE (1988), BraUNGART (1988), STAGL (1990), HonMANN (1992); GeTnER (1992); zu Kunstgeschichte und Architektur vgl. WARNKE (1996) und STURMER (1980). Die Hoforschung der 1990er Jahre schloB in ihrer politik und sozialgeschichtliche Analyse die Frage nach dem Zeremoniell ein - AsCH/BIRK (1991), HOLENSTEN (1991), KungeNSMTH (1993) - und knupfte damit an die frühe Arbeit von PLODECK (1972) an. Die Bände von BERNS/RAHN (1995), BAUER (1993), MULLR (1995) und PARAVCINI (1997) arbeiteten den interdisziplinären Forschungsstand auf und stellten Systematisierungen und auch ganze Fragenkataloge vor. Auf dieser für interdisziplinäre Ansätze sehr offenen Grundlage wurde Zeremoniell nunmehr auch stärker historisiert, wobei Zeremoniell im Spannungsverbältnis von Politik, Recht und Ritual verstärkt als Problem von Kommunikation und Körperlichkeit konzeptionalisiert wird: KOLOCH (1996), WINTERLING (1996), ARNADE (1996), STOLLERG-RILINGER (1997), PARAVICINI BAGLIANI (1997),

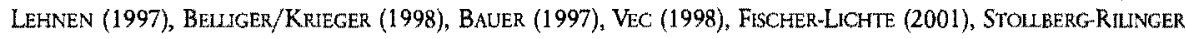
(2001), SCHENK (2003), WIRTH (2002). Zur Literatur 1995-2000 vgl. HIRSCHBIEGEL (2000).

PDF mit freundlicher Genehmigung zur Verfügung gestellt von Konstanzer Online-Publikations-System (KOPS)

URN: http://nbn-resolving.de/urn:nbn:de:bsz:352-opus-105707 URL: http://kops.ub.uni-konstanz.de/volltexte/2010/10570 
gefangen gewesen sei. ${ }^{4}$ Vielmehr sind gerade im Verlauf der Debatte über die Frage der Rationalität bzw. Sinnhaftigkeit höfischer Lebensformen ${ }^{5}$ die konstitutive Bedeutung zeremonieller Verhaltensformen für die frühneuzeitliche Gesellschaft und auch das Zeremoniell als ernstzunehmender Forschungsgegenstand anerkannt worden.

Die sehr komplexe neuere Forschungslage hat indes auch deutlich gemacht, daß der Terminus Zeremoniell/Hofzeremoniell ungeachtet etymologischer Annäherungen und der Heranziehung oft zeitgenössischer Definitionen und Sammlungen zeremonieller Akte noch keinen Gegenstand konstituiert, sondern allenfalls ein Forschungsfeld umreißt. Hofzeremoniell bzw. Zeremoniell war, wie Miloš Vec zeigt, in der Konzeption besonders der frühneuzeitlichen Autoren häufig ein normativer Lösungsansatz für das Problem gesellschaftlicher Ordnung; von daher versteht sich auch der enge Bezug des Zeremoniells zu Recht und Verfahren, den besonders Barbara Stollberg-Rilinger betont. ${ }^{6}$ Wenn aber einerseits konstatiert wird, das Zeremoniell habe am Hof ,alle Lebensbereiche" geformt, ${ }^{7}$ andererseits die Historizität des Begriffes hervorgehoben und auf einem davon abgehobenen analytischen Begriff bestanden wird, ${ }^{8}$ scheint Bedarf an einer breiteren Diskussion zur Klärung von Terminologie und Theoriebildung gegeben zu sein.

Eine vermittelnde Position kann an einem Konzept von Kommunikation unter Anwesenden (Interaktion) ansetzen, das darauf verweist, daß in Interaktion Sinn zugeschrieben wird und daß die damit verbundene Semiotisierung von Situationsmerkmalen ein grundsätzlich offener Prozeß ist - so daß sich an Interaktion in der Tat außerordentlich dichte „Zeichensysteme" festmachen können, die dafür sorgen, daß ,Verhalten [...] mehr und mehr nur noch als Kommunikation betrachtet, analysiert und verfeinert wird “9 und der Eindruck umfassender Geltung entsteht. Problematisch an der durchgreifenden Semiotisierung von Verhalten wird dann jedoch die Frage, für welche Beobachter welche Zeichen Informationswert gewannen, inwieweit Individuen, Gruppen, Gesellschaften daraus Konsequenzen zogen und wie hierbei historischer Wandel zu beobachten ist. Es lassen sich dann Fragen wie die nach der Bedeutung der Medialität der Dokumentation für diesen Wandel stellen und Referenzen diskutieren, die den Begriff des Hofzeremoniells trennscharf machen. ${ }^{10}$ Ein möglicher Ertrag wäre beispielsweise eine Fortführung der Debatte um die Krise des Zeremoniells, ${ }^{11}$ die sich aus einer solchen Perspektive als Krise von Interaktion als Kernsituation sozialer Reproduktion darstellt. Die Hofbälle des 19. Jahrhunderts beispielsweise waren um vieles minutiöser organisiert und insofern „zeremonieller" als die des 18. oder gar die Faschingsfeste des 17. Jahrhunderts - die Relevanz der Interaktion aber war vor dem Hintergrund des Vordringens der formalisierten Kriterien funktionaler Differenzierung auf ein anderes, sozial folgenärmeres Spektrum verwiesen.

4 RiLl (1992) 7, 333.

s Vgl. hierzu besonders KrUedener (1973), Ehalt (1980), Ehalt (1977), WinTERLING (1986), BaUer (1993), DUINDAM (1994).

6 Vec (1998), STOLlberg-Rilinger (2001), Hengerer (2001).

7 STANGL (2001) 14.

* HofmanN (1985) 24, beklagt „die verfehlte Gleichsetzung der Etikette mit dem Gesamtphänomen des Hofzeremoniells“. Beispielhaft für den Ausweg in der Form subtiler Abgrenzungsvorschläge STAGL (1990).

9 Zum Interaktionsbegriff vgl. KIESERLING (1999), zitiert nach LUHMANN (1993) 98 (Hervorhebung im Original); zur semiotischen Dimension vgl. RAHN (2002), ZACHARIN (2000).

10 Zur Medialität vgl. Duindam (2001a) und RAHN (2002) 25-28. Als Systemreferenz, von der aus Hofzeremoniell sich von anderen Formen von Verhalten abgrenzen ließe, habe ich an anderer Stelle den Hofstaat als Organisation vorgeschlagen, im Sinne Stollberg-Rilingers also das „Binnenrecht ${ }^{\star}$ des Hofes, HengerER (2001). Zu dem, was an Relationierungen alles möglich ist, vgl. VEC (1998) 63-79.

"JaHn/RaHN/SCHNITZER (1998), VOCELKa (2001), HOLENSTEIN (1991). 
Vor diesem Hintergrund sowic in Anbetracht des Forschungsstandes zum Zeremoniell des Kaiserhofes ${ }^{12}$ erschien es angemessen, in diesem Band auf die Quellen zur Geschichte des Zeremoniells im Bereich der Habsburger hinzuweisen, deren Hauptbestände im Haus-, Hofund Staatsarchiv (HHStA) in Wien bewahrt werden. Unter diesen Quellen hat das Zeremonialprotokoll eine zentrale Stellung, weil es als chronologisch fortlaufende schriftiche Fixierung einer systematischen Selbstbeobachtung des Hofes zur Sicherung des Gedächtnisses an den Ablauf spezifischer Geschehnisse konzipiert, institutionell beim obersten Hofamt verortet und mittels einer zuständigen Stelle auf Dauer gestellt wurde. Dies läßt sich von anderen, teilweise weitaus älteren, umfangreicheren und nicht selten detailreicheren Beständen, wie sie etwa in den Älteren Zeremonialakten im HHStA zusammengefaßt sind, nicht sagen. Von daher steht das im Jahr 1652 begonnene und bis zum Ende der Monarchie fortgeschriebene Zeremonialprotokoll im Zentrum der folgenden Ausführungen. Ein Überblick über die zentralen Bestände zum Zeremoniell der Habsburger vor allem im HHStA schließt sich an.

\section{Zum sogenannten Zeremonialprotokoll (Protocollum Aulicum in Ceremonialibus)}

Entstanden ist das Zeremonialprotokoll im Jahr 1652 als Ergebnis des Versuchs einer grundlegenden Reform des kaiserlichen Hofstaats. ${ }^{13}$ In den vorangehenden Jahrzehnten war der Hofstaat einem tiefgreifenden Wandel unterworfen gewesen, der auch zeremonielle Probleme mit sich brachte; seit Anfang Juni 1650 waren zudem rasch nacheinander mehrere erfahrene Inhaber oberster Hofämter verstorben, welche die teilweise erheblichen Differenzen zwischen den überkommenen und vielfach veralteten Normen des Hofstaats und einer partiell sehr flexiblen Praxis zu handhaben imstande gewesen waren. Nicht lange danach, im Frühjahr 1651, setzte Kaiser Ferdinand III. eine Kommission mit dem Auftrag ein, Pläne für die Wiederherstellung der früheren Hofordnung auszuarbeiten und diese an die neuen Verhältnisse anzupassen. Die Kommission war hinsichtlich der möglichen Erträge einer ebenso modernisierenden wie restituierenden Reform indes skeptisch und beschränkte sich auf eine geringfügige Überarbeitung der vorhandenen Instruktionen. ${ }^{14}$

12 Erst unlängst wurde darauf aufmerksam gemacht, daß die grundlegenden Fragen nach Herkunft, Tradierung und Modifikation des burgundischen Zeremoniells am Kaiserhof des 16. und 17. Jahrhunderts und seiner Uberformung durch das spanische Hofxeremoniell im 18. Jahrhundert meistenteils unbeantwortet sind, bes. HormaNNRANDALL (1995) 156, PARAVICNa (1991), Von daher bedürfen die um ihres Bemühens um eine umfassende Deutung willen verdienstyollen Darstellungen wie die von EHALT (1980) und BAUER (1993) einer Aktualisitrung. Auf der Ebene von Studien zu einzelnen Anlässen bzw, zu Serien zeremonieller Akte liegen zahlreiche Studien wor, wobei wegen der hier gebotenen Küze keine Bibliographie, sondern nur einige wenige Beispiele genannt werden können: Wahlen, Krönungen, Erbhuldigungen: WANGER (1994), REUTER-PETTENBERG (1963), HATENHAUER (1995), PUCHL (1954); Tafelzeremoniell: Hastnger (2002), BAST//HEISS (1995), LOWENSTEIN (1995); Taufen und Hochzeiten: VockikA (1976), STOCKEuE (1982); Tod und Bestattung: HAWLK-YAN DE WATER (1989), STANGL (2001), BRXX (1973), Kirchliches Zeremoniell: KovAcs (1979), vgl aus dem Bereich der darstellenden und bildenden Kunst und Atchitektur: SOMMER-

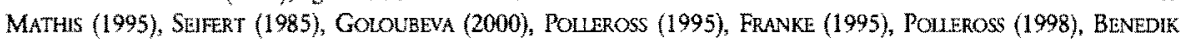
(1997). BENEDK (1997a), GRAF (1997). Vor dem Hintergrund der interdisziplinären Debatte zum Zeremoniell stellen sich viele Fragen indes neu, während unterhalb der Ebene der Großereignisse insgesamt noch eher wenig erforscht ist, vgl. VOCELKA (2001), VOCEIKA/HELER (1998), fur Frankreich CHATENET (2002).

13 Vgl. zum folgenden knapp: Žolger (1917) 155, SOMMER-MATHIS (1995) 514-515, DUINDAM (2001) 194; autührlich: MENCIK (1899) 459-462, DUINDAM (2001a) 375-377, HENGERER (2002) 260-264.

I4 HHStA, Obersthofmeisteramtsprotokolle [OMeA] SR, K. 72, Konv. 121, unfoliiert, verm. Februar 1651: „weilen die alte gute Hoffordnungen zimblich zerfallen, und underschidliche Unordnungen eingeschlichen, thails sich auch der status selbst geändert", wurde beschlossen, „daß demnach de remedio zugedenckhen und dahin zuse- 
In diesem Rahmen wurde auch die Instruktion des zum neuen Obersthofmeister ernannten Fürsten Maximilian Dietrichstein (gest. 1655) erörtert, zu dessen Pflichten die Teilnahme an zeremoniellen Akten gehörte. Für die Eingrenzung der Präsenzpflichten fand die Kommission zwar zu einer handhabbaren Klassifizierung zeremonieller Akte. ${ }^{15}$ Eine definitive Regelung der besonders problematischen Eingeleitung fremder Fürsten hingegen lehnte sie mit einer dreistufigen Argumentation ab: Es sei wegen der Vielzahl der Fälle nicht möglich, klare Regeln zu finden; wegen der Zahl der - zudem partiell umstrittenen - Kriterien seien auch die Fälle nicht vorhersehbar; und schließlich sei eine Instruktion kein geeigneter Ort für eine schriftliche Regelung. ${ }^{16}$ Damit betonte die Kommission neben der Komplexität zeremonieller Probleme auch die nicht minder bedeutsame Frage der angemessenen medientechnischen Form für die schriftliche Behandlung des Stoffes. Inhaltlich verwies die Kommission Dietrichstein auf die jeweilige kaiserliche Einzelentscheidung.

Bei der Frage der Dokumentation kam sie ihm jedoch entgegen und regte die Führung eines Zeremonialprotokolls an, das die „casus" schildern sollte und später als Referenz dienen könnte. ${ }^{17}$ Dieses „Ceremonial" sollte derjenige Sekretär führen, den Dietrichstein für die Aktenführung in seinem Obersthofmeisteramt erbat ${ }^{18}$ und von dem die Kommission meinte, daß „Ihme auch die Ceremonialia aufgetragen werden möchten“. ${ }^{19}$ Im September

hen seÿe, wie diese Unordnungen reparirt, und solche, sowohl zu der alten Ordnung und observanz, sovil dieselbe ad modernum statum tauglich, reducirt, also auch zumahlen, daß Werkh nach den iezigen läuffen eingerichtet und formirt werden möchte." Die Gutachten wurden in dem zeitnah angelegten Kopialband mit über 40 Instruktionen aufgenommen (HHStA, Zeremonialakten [ZA] SR 10), so das Gutachten über die Reform von 1651 mit besonderer Berücksichtigung des Obersthofmeisteramtes (pag. 368-380), des Obersthofmarschallamtes (pag. 381-389) und des Oberststallmeisteramtes (389-392) mit der Feststellung, daß keine kaiserliche Instruktion vorliege (pag. 389-392, 389). Vermutlich wurden auch aufgrund dieser Sitzung die vorgeschlagenen Verzeichnisse des Hofstaats Kaiser Ferdinands III. angelegt (HHStA, OMeA SR 186 und 187). Einen Hinweis darauf, daß um 1650 das Zeremoniell bei Hof ohne breitere personelle Kontinuität nicht mehr zu überblicken war, gibt die etwa zeitgleiche Anlage eines Zeremonialbuches zur Nuntiatur Pannochieschi (1652-1658) seitens der Nuntiatur, das ebenfalls den Anfang einer Reihe von besonderen Zeremonialrelationen machte, vgl. dazu MEISTER (1891) und HENGERER (2002) Kap. III.2.a.

${ }_{15}$ HHStA, OMeA SR, K. 72, Konv. 121, unfoliiert, verm. Februar 1651. Der Obersthofmeister sollte gemäß seiner Instruktion in der Lesart der Kommission präsent sein bei „allen solenniteten“, bei denen der Kaiser zugegen sei, „es seÿe zu Kirchen, Einritt, Ladtschafften und andern dergleichen offenen acten“; dies sollte künftig jedoch „nit ordinarie, auf die gemeine Kirchengäng, Propositionstäg, Lehenstäg, Lehensverleihungen und dergleichen, sondern nur auf die Zeit und Täg der Crönungen, Huldigungen, Churfürstlichen Tägen, offenen bancheten am Kä̈l: Hoff, wie auch beÿ Verleihung Churfürstlicher Lehen, und da dern Persohnen selbst zugegen sein, und andern gleichmässigen offentlichen actibus" gelten.

${ }^{16}$ HHStA, OMeA SR, K. 72, Konv. 121: „Wan nun aber propter multipliticationem casuum fast unmüglich, eine gewiße regel disfals zu sezen, sondern, Wie die fürsten selbst, in multiplici differentia constituirt sein, und under denselben Fürsten selbst, diversa ratio aintweder der Verwandtnus, Standts, mehrern oder wenigern Confidenz, wie auch des Orths, in- und außerhalb der Residenz, und anderer Umbstände halber ist; und es einen großen absaz darmit hat, also khünen auch nit alle casus \& circumstantias vorzusehen, und etwas aigentliches, zuemahlen in ein instruction einzubringen sein."

17 HHStA, OMeA SR, K. 72, Konv. 121: „[...] doch würde sehr vorträglich sein, wan man etwo ein gewißes Ceremonial aufrichten thätte, worin, sovil müglich, die casus mehrers specificiert werden möchten, darnach man sich in fürfallenden occasionen khünfftig reguliren könte“.

${ }^{18}$ HHStA, OMeA SR, K. 72, Konv. 121: „[...] damit alle Ambts sachen registrirt, prothocolliert, in Ordnung erhalten, und man künfftig in allem gute nachricht von dem fürgangenen geschäfften haben, auch alles desto ordentlicher hergehen möge“.

${ }^{19} \mathrm{Vgl}$. die Anregung Gundakers von Liechtenstein, eines Obersthofmeisters Kaiser Ferdinands II., der ähnlich wie Dietrichstein im Jahr 1652 bereits 1625 eine ausführliche Erläuterung seiner Instruktion erbeten hatte, welche bei der Reform von 1651 als fortgeltende Zusatznorm approbiert wurde. Liechtenstein hatte nach seiner Amtszeit im Jahr 1641 geraten, man solle bei Hof einen Zeremonienmeister bestellen und „ein Prothocoll halten, wie es zu Hoff und anderstwo gehalten worden mit denen terminis ceremoniarum, damit man ein Nachrichtung haben könne 
1652 setzte die erhaltene und bis zum Ende der Monarchie fortgesetzte Protokollierung ein und überführte die Komplexität der unvorhersehbaren "casus" als Narrativ ins Archiv. ${ }^{20}$

Inhaltlich war das Protokoll freilich von Anfang an nicht auf die Dokumentation derjenigen Detailfragen, die für seine Entstehung Anlaß gegeben hatten, beschränkt. Vielmehr bezog es sich im wesentlichen auf Elemente der nicht alltäglichen Interaktion des Kaisers und/oder seinet Angehörigen mit Dritten. ${ }^{21}$ Abstrakt läßt sich der Gegenstand des „Ceremonials" in der Terminologie der Kommission in etwa mit den Begriffen „solenniteten " und „offentliche actus", soweit sie nicht "gemein" waren, umreißen. ${ }^{22}$ Konkret wurden im ersten, die Jahre 1652 bis 1659 umfassenden paradigmatischen Band zahlreiche derartige Großveranstaltungen beschreiben, so etwa: Ankunft, Einzüge, erste Audienzen von Gesandten, Botschaftern, Fürsten und Kurfürsten, Visiten, Revisiten, Essen, Abschiede und Abreisen - Wahlen, Krönungen und Erbhuldigungen, Land- bzw. Reichstage der verschiedenen Herrschaftszusammenhänge - Reisen, Reiserouten, Empfânge und Einzugsordnungen des Dynasten - Fürstenstand serhebungen und Belehnungen - Geburten, Taufen, Tod, Aufbahrung, Begräbnis, Exequien (HHStA, ZA Prot. 1). Je höher der Rang der Personen war, mit denen die Dynasten in Kontakt kamen, desto detaillierter war die Dokumentation, was zur Erweiterung der beschriebenen Anlässe über den Kernbestand hinaus und in diesem Rahmen zur Einbeziehung ,gemeiner' Veranstaltungen führen konnte.

Von daher findet sich bei der starken Betonung dessen, was die Zeremonialwissenschaft als ,Staatszeremoniell ${ }^{\star}$ bezeichnete, ${ }^{23}$ auch das Zeremoniell des Hofes in den Zeremonialprotokollen, wobei aus der Perspektive der richtigen Gestaltung der Großveranstaltungen etwa die umstrittenen Ränge der Hofdamen häufiger dokumentiert wurden. Für die innere Ordnung des Hofstaats elementare Ereignisse sind als ,gemeine ${ }^{t}$ Veranstaltungen eher selten, dann vornehmlich bei höchstrangigen Hötlingen beschreiben: ${ }^{24}$ "In Monath Junij ist nichts denkhwürdiges fürgangen", heißt es im Jahr 1654, obschon ein neuer Geheimer Rat seinen Eid leistete ${ }^{25}$ - und solche Monate waren nicht selten.

Es ging also um „denkhwürdiges", und mit diesem zugleich differenz- und typenorientierten Ansatz fiel dasjenige aus dem Dokumentationsbereich heraus, dessen Ablaufform zum unproblematisch erscheinenden höfischen Alltag gehörte oder auf anderer Ebene do-

bey denen Solleniteten und Ambasciaten“, MrTS (1908) 114; zu Liechtenstein vgl, ausfühlich WINKELBAUER (1999); knapp in diesem Zusammenhang: MENCiK (1899) 461. Möglicherweise bezog man sich auf diesen Vorschlag.

20. Die genauen Umstände der Entstehung der einzelnen Einträge im Zeremonialprotokoll sind bislang noch kaum untersucht worden. Dazu gehört leider auch die Frage, wie genau der jeweilige Verfasser zu seinen Informationen $\mathrm{kam}_{1}$ ob als Teilnehmer (mit welchem Standort), ob als Kompilator von Erzähltem und/oder schriftich Vorgelegtem; hier wiederum wäre die Frage zu beantworten, inwieweit die Berichte eventuell eher von den Planungsakten abhängig sind als vom Geschehensablauf. Zu semiotischen Problem der Verschriftlichung vgl. RaHN (2002) 27f. Bekannt ist, daß zunächst Konzepte verfertigt wurden und nach einem wiederum im wesentlichen unbekannten Uberarbeitungs- und Genehmigungsverfahren überttagen wurden, Kraus (1937) 297.

${ }^{21}$ Die Zuständigkeit für zeremonielle Fragen lag im wesentichen beim Obersthofmeister: Žo.GER (1917) 66-69, 77; KRAus (1937) 276f; SOMMER-MATHS (1995) 515; zum Amt bis heute grundlegend: MExCIK (1899).

22 Die Protokolle waren, wie KRAUs (1937) 297 hervorhebt, keine Geschäftstagebijcher des Zeremonialdepartements, auch keine Registerbände: „Sie sind selbständige Aufzeichnungen über alles, was an jedem Tag um die Person des Herrschers öffentlich bei Hofe zugeht und geschieht. Der kaiserliche offentliche Kirchgang, der Empfang von Gesandten und Würdenträgern, die Belehnungen, die öffentlichen Trauern und Feiern, an denen der Kaiser teilnahm, seine Reise usw.: all dies bildet den Inhalt der Zeremonialprotokolle."

as Vi. Vec (1998) 71 .

24 So etwa die Neubesetzung von Oberstkämmerer-, Oberstallmeister-y, Oberstjäger- und Oberstfalkenmeisteramt im Februar 1655, HHStA, ZA Prot. 1, 472.

35 So der Geheime Rat Gonzaga, vgl. SCHWARz (1943) $236 f$. und SiENEI1. (2001) 82-84. 
kumentiert wurde. ${ }^{26}$ Auf der anderen Seite wurde die Dokumentation der außerordentlichen

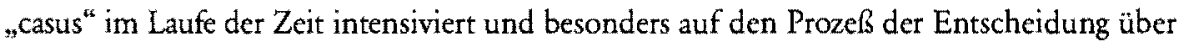
die Gestaltung solcher Akte ausgeweitet: Referate, Voten, Beschlüsse und Sitzungen über die Gestaltung von Veranstaltungen und in diesem Zusammenhang auch Sitzungen des Geheimen Rates wurden aufgenommen. Zugleich wurden die Schilderungen vor allem in der ersten Hälfte des 18. Jahrhunderts detaillierter. Welche Phänomene aber unter welchen Umständen dokumentiert wurden, was den Stellenwert eines aufzeichnungswürdigen Ereignisses bzw. Details bekam, ist eine bislang noch kaum reflektierte Frage.

Un vor diesem Hintergrund zumindest einen näheren Einblick in die Inhalte des Protokolls zu geben, möchte ich im folgenden zunächst Inhalte der Dokumentation für das Jahr 1665 nennen, das mit Ausnahme der Übernahme Tirols durch die Habsburger Hauptlinie cin durchschnittliches ruhiges Jahr repräsentiert und für die Führung des Protokolls besonders deshalb aufschlußreich ist, weil es zeigt, daß auch andere im Archiv nachsahen, wie man zeremonielle Anlässe gestalten konnte bzw, wollte.

\section{ZA Prot. 2, pag. 1117-1262 (1665):}

Im Januar sei „nichts sonders bej Hoff vorbey gangen", lediglich sei die Landtagsproposition

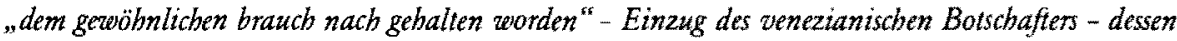
erste Audienz bei Kaiser und Kaiserinwitwe - Tod und Bestattung des Obersthofmeisters Portia - Eid und Introduktion des neuen Oberstbofmeisters - Kriegspräsidentenvereidigung - Deutscbmeisterleben - bischöflich Olmützer Lehen - Toisonverleibung - Audienz des Botschafiers zur Pforte mit Zugordnung - Liste von Geschenken an türkische Amtsträger - Audienz für den siebenbürgischen Gesandten - Ankunft und Audienz einer moskowitischen Gesandtschafi - deren Konferenz inkognito mit kaiserlichen Amtsträgem - Einzug des türkischen Großbotschafters mit Zugordnung - dessen Audienz beim Kaiser - Geschenke des Großbotschafters - im Juli: „nichts denkbroürdiges in curialibus bej Hoff vorgefallen" - Exequien für Erzberzog Sigmund Franz von Tirol, Kammerklage - katserliche Wallfabrt nach Mariazell - Reise zur Erbhuldigung nach Tirol - Darlegungen des Erzbischofs von Salzhurg bzgl. des Zeremoniells des Treffens unter Bezug auf Treffen von Kaiser und Erzbischof 1619 und 1622 - Treffen von Kaiser und Erzbischof, Audienz, Eingeleitung und Einzug des Kaisers in Salzburg mit Zugordn ung nach Beschluß in der Sitzung des Gebeimen Rates (ZA Prot. 2, pag. 1206) - Tagreisen von Wien nach Salzburg - Aufenthall in Salzburg, Essen, Messen, Komödie, Feuerwerk, Besichtigungen, Abschied - Reiseverlauf, Empfänge untereegs - Einzug in Innsbruck mit Zugordnung - sehr ausfübrlich: Aufentbalt in Innsbruck im Oktober, insbes. Erbbuldigung - Rückreise nach Wien - Einzug des Nuntius - dessen erste Audienz bei Kaiser und Kaiserinwitwe - bischöflich Passauer Lehen - Exequien für den spanischen König.

Um weiter einen Eindruck von der Textur einzelner Beschreibungen zu geben, folgen partiell aufeinander verweisende Beschreibungen gleichartiger Geschehnisse.

Ankunft der verwitweten Kurfürstin von Bayern, einer Schwester Kaiser Ferdinands III., Prag, 29. September 1652 (ZA Prot. 1, pag. 2-4): ${ }^{27}$

"Wie dan eben diesen tag als den 29. an einem Sontag und fest des bojl. Emzengels Micbael umb 3 Ubr Nacbmittag, Ibr Kä̈. Mä̈. mit der Kä̈serin, und der zu Hungam und Böbaimb Königl.

26 Vgl. das Eidbuch HHStA, Hs. Weiß 23/706, das auch zeremonielle Aspekte dokumentiert.

${ }^{27}$ Die Zitienweise orientiert sich mit geringfügigen Abweichungen bei engerem Festhalten am Buchstabenbestand sowie teilweiser Auflösung der Abkürzungen an WINKELBAUER (1999) $19 \mathrm{f}$. 
Mä. ${ }^{\text {ten }}$ in einer Carroza bej samben siezent, in beglaittung des Spanischen Pottschaffers, und der bochen Ministri, gehaimben Rathe, Camerem und Cauagliren, Ibrer Churfürth. Durchlaucht ungefehr mit 40 Carrozen zu 6 Rossen in nach volgender ordnung entgegen gefahren.

Als Erstlich sein 2 Compagnien zu Roß, won den Monticucolischen Regiment geritten, darauf die wägen der Cauagliren, Camerer, bochen Ministri und gebeimben Räthe gevolgt, Nach denen die königl. und sodan die kaj. Camerer in denen Hofferägen, und dan Ibrer kay. Maj. Obr. Hoffmaister, Ibr Fürstl. gn. von Dietrichstain, Verers der obr. Stallmaister in ein Hoffwagen, und nach Ibne der Spanische Pottschaffter in seiner Carroza gefahrn. Gleich darauf Ihrer Kaÿ. Mt. Wagen, nach welchen der Fürst Piccolomini als Guardi Haubtman mit der ganzen Hartschier Guardi, sambt Hörpauckhen und Trombetten gevolgt, So dan seint allererst der kaj. Hoff Damen, wie auch der Camerdiener Waigen gangen.

Als nun Ihr Kay. Mä. in solcher ordnung fort gezogen, und wngefehr gegen den Stern über angelangl, ist baldt darauf der Frauen Churfürstin wagen berfür khomen, und wie Sje vast hundert schritt von Ihrer kay. Maj. wagen gevesen, seint Ibr Durchlaucht abgestigen, und neben denen bey sich gebabten Cauagliren und Damen zu Fueß dem kay. wagen zuegangen, darauf auch baldt Ibre Maj.en Kaÿser, Kaÿserin, und König abgestigen, und mit Ihren comitat, Ihrer Churfürtl. Durchlaucht entgegen gangen, und Sye aneinander empfangen, auch nach beschechner complimentinung. Ibre Majy." der Kajser, Kaj̈serin und König der Frauen Churfürstin Cauagliren und Damen, wie in gleichen Ihr Durchlaucht denen kaj. und königl. die Handt gebotten.

Nach welchen Ihre Maÿ." widerumb aufgesessen, und die Frau Chur Fürstin mit Ithen in wagen genohmen, die da neben Ibr Mt. den König zur linggen handt gesessen, die kaÿ. hoche Ministri und gehaimbe Rätbe, baben die Chut Bayrische in lhre Carrozen zu sich genobmen, und undereinander getheilt, so dan auch in der ordnung wie in der hinaus fabrt widerumb zu ruckb gegen der Statt gefahren, und als man etwas binzue genahet, ist auf denen Pasteyen ein Salve aus 83 Stuckh geschüz geben worden, auf den Ratschiner Plaz aber vor den Schloßh ist ein Regiment von 5 Fendl in einer Squadron, und gegen über 3 compagnien zu Roß gestandten.

Wie nun Ihre Maj." und Durchlaucht in das Schloß ankhomen, und bej der grossen Haubt stiegen abgestigen, hat entzreischen besagtes Regiment und die Reutterÿ ein Salve geben."

Ankunft des Kurfürsten von der Pfalz, Prag, 5. Oktober 1652 (ebd., pag. 7):

"Nachmittag umb 2 Uhr, seint Ibre Kay. und Königl. Maj." in einen zeagen bejsamben, Ibrer Chur Fürstl. Durchlaucht dem Pfalz graven entgegen gefahm, eben mit den comitat, und so weit, auch sonsten obne underschiedt, so woll des empfangs als in allen andern wie es mit Ihr Durchlaucht der Chur Fürstin in Baÿm gehalten, wie nit weniger auch von 80 Stuckb geschüz, und vorbedeuten Squadron, das Salve geben worden."

Ankunft des Kurfürsten von Trier, Prag, 6. Oktober 1652 (ebd., pag. 8f.):

„Den 6. Nachdem Ihr Kä̈. Maj. in gewensse erfabrung khomen, das Herr Churfürst von Tÿ̈r nit weit won Prag auf der Post angelangt haben Sje demselben ein Leibwagen, sambt andem 2 Wägen entgegen geschickbt, wie nit weniger Ibr Kä.. Maÿ. auch selbst, demselben entgegen gefahm, und es mit dessen einbeglaitung in allen, wite mit denen andem Herm Chur Fürsten balten lassen."

Ankunft des Kurfürsten von Sachsen, Prag, 4. Oktober 1652 (ebd., pag. 30-33):

„Einzug Ihrer Churfürstl. Durchlaucht zu Saxen in Prag.

Als Ihr Durchlaucbt der Chur Fürst sambt des Cbur Prinzens Dt. und dero Hoff Statt bey den Perg Ziegenruckb ankhomen, und der Vortrab über die böche fort gangen, haben Ibr Dt. auf den flachen Veldt bey den Vor Werkb Subtball, Ibro fürstl. Gn. von Liechtenstain gebörig 3 Vürtl Meill reegs 
von Prag gehalten, daselbst Sÿe von den Wagen abgestigen, und Ibrer Käj. Maj̈. ankbonffi erwarthet, und nachdem Sj̈e sich ein Zeithero aufgehalten, kbäme ein Courrit, welcher die nache ankbofft Ibrer Kajy. Maj. vermeldet, darauf sezten sich Ibr Churfürstl. Dt. wiederumb zu Wagen, sambt Ihr Dt. den Chur Prinzen, zur rechten, und Herzogen Julius Heinrich zu Saxen zur linggen Handt ruckbwerts, verers gieng alles in seiner guetten ordnung auf der Strassen forth, Ibrer Churfürstl. Dt. geheimbe Räthe, Cauagliri und officir, giengen nache beÿ dero Leib Wagen ber, In deme kbonten [!! Sÿe vast nachent zu Ihr Käj. Mäj. wurden die Hörpaukhen geschlagen, und die Trombetten geblasen, und begabe sich der Herzog von Saxen, dan Ibr Dt. der Chur Prinz, und volgents Ihr Chur Fürstl. Dt. von dem Wagen, darauf so dan Ibr Königl. und entlich Ibr Kä̈. Mä̈. nach denen Ministris abgestigen, Ibr Dt. der Chur fürst und Chur Prinz giengen Ibren Kaj.. und Königl. Maj.. ${ }^{\text {en }}$ auf die 30 schrütt, Ibre Majy. ${ }^{\text {en }}$ aber Ibren Dt. auf die 8 schrütt entgegen, und baben Ibre Kaj. Majy. erstlich den Chur Fürten und bernach den Chur Prinzen empfangen, volgents empfiengen gleichsfabls Ibr Königl. Mä̈. erstlich den Chur Fürsten, und nachmabls den Chur Prinzen, baben auch Ibre Mä̈. mit entdeckthen Haubt auf Ibrer Dt. gethanne Redt geantwortt, Verers baben Ibre Maj.. den Saxischen Cauagliren und Ministris und nachmabls ingleichen Ibre Dt. denen Kaj. und Königl. Ministris die Handt zu Khüssen geben, die kaj̈. Reutterey und die ganze beglaittung bielte den Sachsischen zur linggen, die aber zur rechten Handt, Nach beschechnen Empfang sezten sich Ihr Kaj. Mä. widerumben in dero Leibwagen, und Hüessen Ibr Chur Fürstl. Dt. zu Ibnen sizen, welche aber sich dessen gewaigert, bis Ibr Königl. Mä̈. zuvor aufgesessen, und die ober Handt zuruckbs eingenobmen, als dan sezten sich Ibre Chur Fürstl. Dt. zu der Königl. Mä̈. linggen Handt, Ibr Dt. der Chur Prinz aber sezte sich unden an in den Schlag, und vermainte aus Ebr erbiettung mit entblösten Haubt zu fabren, wurde aber von Ibr Kä̈. Mä̈. $z u$ bedeckhen instentig vermahnt, [...] und nach dem der kaÿ. Leib Wagen beÿ Spaten abent sich zum Scbloß genabet, würden alsobaldten 80 Stuckh geschüz losgebrendt, darauf von einen Regiment zu Fueß, welches in seiner Kbrüegsordnung mit gerübrten Spill auf den Plaz under den Wabl bielte, ein Salve geben worden, und wabr bej disen Völckbern vorüber, bin fübran der Einzug zum Staub Pruggen thor, nach den Ratschin volgenter gestaldt genobmen [...]. "(Es folgt eine Einzugsordnung.)

Ankunft des Kurfürsten von Brandenburg, Prag, 15. November 1652 (ebd., pag. 41):

„Den 15. seindt Ihr Kä̈. Maj̈. sambt Ihrer Majy. den König, dem Herrn Chur Fürsten von Brandenburg entgegen gefabrn, so weit und solcher gestaldt, wie dem Chur Fürsten von Saxen, weülln Chur Brandenburg eben auf disen weeg ankbomen.

(In sizen, gehen und beglaitten, hat auch der König die rechte Handt behalten, bë̈ den Empfang hat es starckh geregnet.)"

\section{Zentrale Bestände zum kaiserlichen Hofzeremoniell}

\section{Ehemaliges Hofzeremonielldepartement im HHSt $\mathrm{A}^{28}$}

\section{A. Zeremonialprotokolle - Signatur. ZA Prot., Bd. 1-154, 1652-1918}

"Protocollum Aulicum in Ceremonialibus“: Bd. 1: 1652-1659, Bd. 2: 1660-1674, Bd. 3: 1671-1681, Bd. 4: 1681-1691, Bd. 5: 1692-1699, Bd. 6: 1700-1709, Bd. 7: 1710-1712, Bd. 8: 1713-1715, Bd. 9: 1716, Bd. 10: 1717-1719, Bd. 11: 1720-1722, Bd. 12: 1723-1724, Bd. 13: 1725-1727, Bd. 14: 1728-1731, Bd. 15: 1732-1734, Bd. 16: 1735-1738, Bd. 17: 1739-1740, Bd. 18: 1741-1742, Bd. 19: 1743-1744, Bd. 20: 1745-1746, Bd. 21: 1747-1748, Bd. 22: 1749-

28 Siehe dazu Kraus (1937) 295-300. Abgegeben wurden nach dem Ende der Monarchie v.a. Akten zu böhmischen und ungarischen Krönungen, Landtagen, Huldigungen und Reisen. 
1750, Bd. 23: 1751-1752, Bd. 24: 1753-1754, Bd. 25: 1755-1756, Bd. 26: 1757-1758, Bd. 27 : 1759-1760, Bd. 28: 1761-1762, Bd. 29: 1763-1764, Bd. 30: 1765, Bd. 31: 1766, Bd. 32: 17671768, Bd. 33: 1769-1770, Bd. 34: 1771-1773, Bd. 35: 1774-1780, Bd. 36: 1781-1785, Bd. 37: 1786-1790, Bd. 38: 1791-1792, Bd. 39: 1793-1801, Bd. 40: 1802-1804, Bd. 41: 1805-1807, Bd. 42: 1808, Bd. 43: 1809, Bd. 44: 1810, Bd. 45: 1811, Bd. 46: 1812, Bd. 47: 1813-1814, Bd. 48: 1815-1816, Bd. 49: 1817-1818, Bd. 50: 1819-1820, Bd. 51: 1821-1824.

Danach setzt das sogenannte Zeremonialprotokoll ein, das, ergänzt durch einige Diarien, bis 1917 fortgeführt wird. Erhalten sind zudem Exhibitenprotokolle von 1908 bis 1918 sowie Indexbände (Bde. 94 und 105 sowie Bd. 153: Index zu den Zeremonialakten 1848-1900; Bd. 154: Index zu den Zeremonialakten 1901-1918). Vgl. für die Frühe Neuzeit gegebenenfalls den von Anton Settele verfaßten Bd. 152 (Erläuterungen zum Hofzeremoniell, 15371849)..$^{29}$

B. Konzepte der Zeremonialprotokolle - Signatur: ZerProt., Karton 1-23, 1667 bis Anfang des 19. Jabrbunderts

Die Zeremonialakten wurden „zunächst im Konzept entworfen [...] und danach - nach welchen Stadien der Genehmigung ist noch unbekannt - in Reinschrift niedergeschrieben " . ${ }^{30}$ Diese Konzepte sind teilweise erhalten und wurden später in eine eigene Reihe gebracht. ${ }^{31}$ Es handelt sich dabei - ab Karton 3 in sehr dichter Überlieferung - anfänglich zunächst um Bruchstücke und Auszüge aus dem Zeremonialprotokoll. Die Bezeichnungen wechseln in der Folge häufig (etwa Hofprotokoll in Zeremonialsachen, Zeremonialprotokoll, Protokoll des Hofzeremoniells, Diarium des Hofzeremoniells, Diarium des Hofes, Kaiserliches Hofprotokoll, Hofprotokoll, Protokoll des Hofzeremoniells, Hofprotokoll in Zeremonialsachen, Protokoll über das Zeremoniale des Hofes).

Systematisch benutzt wurden diese Konzepte, soweit ich sehe, selbst in außerordentlich quellennah gearbeiteten Untersuchungen zu Fragen des Hofzeremoniells bislang nicht. ${ }^{32}$ Für die Forschung zur Verschriftlichung des Hofzeremoniells wäre freilich auch ihr Verhältnis zu den Reinschriften aufschlußreich.

Karton 1: 1667-1680, K. 2: 1680-1693, K. 3: 1694-1700, K. 4: 1701-1709, K. 5: $1710-$ 1714, K. 6: 1715-1720, K. 7: 1721-1727, K. 8: 1728-1733, K. 9: 1734-1740, K. 10: 1741, K. 11: 1742, K. 12: 1743, K. 13: 1744, 1746, K. 14: 1747-1748, K. 15: 1751, K. 16: 1752-1753, K. 17: 1754-1755, K. 18: 1756-1758, K. 19: 1759-1761, K. 20: 1762-1765, K. 21: 1766-1771, K. 22: 1772-1783, K. 23: 1784-1798, Bruchstücke Anfang 19. Jahrhundert. Vgl. dazu Hofzeremonielldepartement-Sonderreihe, K. 43 Varia, ZA Protokolle 1801-1817 (Konzepte).

\section{C. Ältere Zeremonialakten - Signatur: ÄZA, Karton 1-95, 1562-1836}

Die Sammlung der sogenannten Älteren Zeremonialakten ist der für die Frühe Neuzeit umfangreichste Bestand, der bei zeremoniellrelevanten Fragen in jedem Falle und im Zweifel eventuell vorrangig konsultiert werden sollte. Für die Zeit vor 1652 sind die Älteren Zeremonialakten ohnedies der erste Referenzpunkt. Anders als die Konzepte der Zeremonialprotokolle wurden die Älteren Zeremonialakten von der Forschung in erheblichem Umfang benutzt, stärker auch als die Zeremonialprotokolle.

29 Zum Registraturdirektor Anton Settele, der im frühen 19. Jahrhundert mehrere systematisierende Bände zum Zeremoniell und dem entsprechenden Archivgut verfaßte, vgl. KRAUS (1937) $282 \mathrm{f}$.

30 Kraus (1937) 297f.

31 Kraus (1937) 297; siehe dort auch Anm. 3.

32 Vgl, etwa die minutiöse Arbeit von STANGL (2001). 
Inhaltlich ist das Spektrum der in diesem Bestand zusammengefaßten Stücke ungeach tet der häufig anzutreffenden Parallel-bzw. Mehrfachdokumentation der auch im Zeremonialprotokoll verzeichneten Themenkomplexe etc. um vieles größer und dichter. Es finden sich diverse Materialien zu Aufenthalten, zu Balletten und Platzverteilungen bei Opernaufführungen, zu Castra doloris, Schlittenfahrten, Fußwaschungen, Namenstagen, Ungebührlichkeiten, Wirtschaften; aber auch Dekrete, Eide, Festdokumentationen, Gesuche, Hoftrauersachen, Instruktionen und Inventare, Korrespondenzen, Leichenbeschreibungen, Personalverzeichnisse und Personalia, Quartiersachen, Rangsachen der Höflinge, Referate, Testamente, Verträge sowie diverse Listen etwa zu Kosten, Teilnehmern oder Reiseetappen und weiteres mehr. Häufig finden sich in den Akten Abschriften aus dem Zeremonialprotokoll (,Extrakte"), häufig Übersichten zu Präzedenzfällen bzw. Vorlagen für die Gestaltung von zeremoniellen Akten, was einen Blick auf die Benutzung des Zeremonialprotokolls im Rahmen der Planung aktueller Veranstaltungen gewährt. ${ }^{33}$ Hinsichtlich der Geschichte dieses Bestandes ist auf die starke Fluktuation der Unterlagen zwischen verschiedenen Beständen hinzuweisen, wobei die Familienakten hervorzuheben sind ${ }^{34}$

Karton 1: 1562-1608, K. 2: 1611-1641, K. 3: 1640-1652, K. 4: 1653-1654, K. 5: $1655-$ 1657, K. 6: 1658-1662, 1664, K. 7: 1663-1666, K. 8: 1667-1673, K. 9: 1673-1673, 1677, K. 10: 1675-1676, K. 11: 1677-1679, K. 12: 1679-1681, K. 13: 1681-1683, K. 14: 1683-1686, K. 15: 1686-1689, K. 16: 1689-1690, K. 17: 1691-1694, K. 18: 1695-1698, K. 19: 16991701, K. 20: 1701-1705, K. 21: 1705-1708, K. 22: 1708-1710, K. 23: 1711-1712, K. 24: 1712, K. 25: 1713-1714, K. 26: 1714-1715, K. 27: 1715-1717, K. 28: 1718-1719, K. 29: 1719-1720, K. 30: 1721-1723, K. 31: 1722-1723, K. 32: 1723-1725, K. 33: 1725-1727, K. 34: 1728, K. 35: 1729-1731, K. 36: 1732-1734, K. 37: 1735-1737, K. 38: 1737-1738, K. 39: 1739-1740, K. 40: 1741, K. 41: 1742-1743, K. 42: 1743, K. 43:1743-1744, K. 44: 1745, K. 45: 1745-1756, K. 46: 1747-1748, K. 47: 1749-1750, K. 48: 1750-1751, K. 49: 1752-1754, K. 50: 1755-1756, K. 51: 1757-1759, K. 52: 1759, K. 53: 1759-1760, K. 54-58: 1760, K. 59: 17611762, K. 60: 1762-1763, K. 61-62: 1763, K. 63-64: 1764, K. 65-66: 1764-1765, K. 67-68: 1764-1766, K. 69-70: 1765, K. 71: 1765-1767, K. 72: 1766, K. 73:1766-1767, K. 74-76: 1767, K. $77-79: 1768$, K. $80-82: 1769-1770$, K. 83: 1770, K. 84: 1770-1771, K. $85: 1771$, K. $86:$ 1771-1772, K. 87: 1773-1774, K. 88: 1774-1775, K. 89: 1776-1779, K. 90: 1780-1781, K. 91: 1782-1789, K. 92-93: 1790, K. 94: 1791-1795, 17. und 18. Jahrhundert, K. 95: 1804-1836, 18. und 19. Jahrhundert. ${ }^{35}$

Es existiert ein älteres Verzeichnis auf Karteikarten, die Arbeit an einem neuen Stückverzeichnis ist weit fortgeschritten (Stand: April 2003).

\section{Neutre Zeremonialakten - Signatur. NZA, Katton 1-485, 1790-1918}

Dieser Bestand führte in gewisser Weise als Fortsetzung der Alteren Zeremonialakten die Sammlung von Akten aus der Zeremoniellabteilung bis zum Ende der Monarchie fort, wobei sich aber eine chronologische Überschneidung mit den Älteren Zeremonialakten ergibt. Angelegt wurde die Sammlung nach einem Rubrikensystem, das sich an einer Klassifizierung

33 Vgl. DUIADAM (2001) 376.

34 KraUS (1937) 296.

33 Die Jahreszahlenangaben geben in der Regel das Jahr des ersten Aktenstückes des Kartons ohne Berücksichtigung der häufig enthaltenen Bezugge auf Präzedenzfalle; die zeitliche Erstreckung versteht sich in der Regel ohne die Berùcksichtigung der zahireichen Beilagen mit Zusammenstellungen ähnlicher, aber späterer Ereignisse. Akten, die auch spätere Zeiträume betrafen, wurden meist nach dem Datum des Anlasses ihrer Entstehung inseriert. 
zeremoniellrelevanter Ereignisse orientierte und daher auch für den Zeremoniellbegriff aufschlußreich ist. ${ }^{36}$ Innerhalb der Rubriken wurde wiederum chronologisch geordnet.

Auswahl von Akten vor 1800: Karton 1: Niederösterreichische Erhuldigung 1792, K. 10: Römische Krönung 1790, K. 11: Römische Krönung 1792, K, 12a, 12b: Böhmische Krönung 1791, K. 13: Böhmische Krönung 1792, K. 17: Belehnungen 1793-1865, K. 18: Hoftrauer, Sterbefalle, Exequien 1792-1798, K. 19: Hoftrauer, Sterbefälle, Exequien 1799-1807, K. 75: Hoffeierlichkeiten etc. 1793-1815, K. 185: Taufakte etc. 1741: Taufe Erzherzog Josephs, 1793-1820, K. 193: Reichshofrat 1793/94, K. 199: Prozessionen 1793-1820, K. 211: Ordensfeste 1793-1811, K. 215: Rangsachen etc. 1794-1805, K. 238: Vermählungen 1795-1799, K. 276: Audienzen etc. 1795-1829, K. 315: Hofreisen etc. 1799-1804.

\section{E. Zeremonialakten-Sonderreibe $=$ Hofzeremonielldepartement-Sonderreibe - Signatur: ZA SR,} Bd. bzw. Karton 1-78, 1625-1918

Die Sonderreihe der Zeremonialakten stellt sich als neu geordneter Rest derjenigen in der Regel aus der Hauptreihe der Zeremonialakten entnommenen Handakten des Zeremonielldepartements dar, welcher bei der Übernahme des Archivs des Zeremonialdepartements durch das Staatsarchiv im 19. Jahrhundert nicht wieder in die Hauptreihe eingereiht wurde, weil er "Merkmale einer berechtigen Sonderung" trug oder sonst „der $W_{1}$ edereinteilung widerstrebte ${ }^{a 3}{ }^{3}$ Es handelt sich somit um für die Praxis wichtige Referenzstücke, die entgegen der sonst üblichen diachronen Ordnung systematisch zusammengestellt wurden.

Auswahl von Akten/Bänden mit Bezug zum Zeitraum vor 1800: Bd. 3: Instruktionen und Hofstaat der Kaiserin Elisabeth Christine, 1740-1750, Bd. 10-13: Instruktionsbücher (1625-1714; 1712-1759; 1749-1789; 1787-1808), Bd. 14: Krönung Franz Stefans' 1745 (=HHStA, Hs. W 525), Bd. 15 und 16: Krönung Josefs II. 1764; Bd. 27: Protokoll der Reise zur Krönung nach Frankfurt 1764, Bd. 38: Hofklagregulierung 1767, Bd. 39: Erneuerte Hofklagregulierung 1767, Bd. 40; Hofklagregulierung 1767 mit Konzepten und Hoftraueranzeigen, K. 41 und 42: Akten zur Hoftrauer, 18. und 19. Jahrhundert, K. 44: Anstellung von Hofbediensteten, 1715-1781 (Stückverzeichnis: AB XII/5/3a), K. 45: Gesuche 1563-1766 (Stückverzeichnis $A B$ XII/5/3b) und Referate vor allem zu Hofreformen des Obersthofmeisters Zinzendorf an Maria Theresia, 1741, K. 47: Varia, 1799-1835, Bd. 50: Vermählung Erzherzog Josephs mit Elisabeth von Bourbon-Parma 1760, Bd. 51: Vermählung Erzherzog Josephs 1760, Bd. 52: Vermählung Erzherzog Leopolds mit Prinzessin Maria Ludovica 1764, 1765, Bd. 53: Vermählung der Erzherzogin Maria Josepha mit König Ferdinand IV. von Neapel 1767, Bd. 54: Vermählung der Erzherzogin Marie Antoinette mit dem Dauphin 1770, Bd. 56: Verwandtschaftstabellen des Kaiserhauses, 1767, Bd. 58: Protokoll der Funktionen bei Hof, beschrieben 1764 durch den Kammerfurier Johann Georg Zinner, Bd. 73: Übertragung von Habsburgern aus Basel und Königsfelden nach St. Blasien, 1770 (Druck), Bd. 74: Tripoliner Gesandtschaft, 1750, verfaßt vom Hofkriegssekretär Schwachheim (Duplikat).

Daneben stehen spätere Aufzeichnungen bzw. Handakten: Settele: Bd. 1: Krönungen, 1638-1690, Vermählungen, 1673-1678, Geburten und Taufen, 1654-1701, Bd. 57: Immerwährender Zeremonialkalender, Fasz. 71: Verzeichnis der Obersthofmeister von 1650-1829,

36 Die ursprüglichen Rubriken I bis XV sind abgedruckt bei KRAus (1937) 299. Hinzugekommen sind: XVI Gardekapitäne, Geheime Räte, Hofdienste, Oberste Hofchargen, Stellvertretung, Truchsessen, XVII. Leibgarden, XVIII. Medaillen, Münzen, Wappen, XIX. Zeremoniell-Vorschriften, Normalien (HHStA, Archivbehelf XII/5).

37 Vgl. KRAUs (1937) 297-300, zur Entstehung 287, 299 (Betreffsonderungen); Zitat: 297. Das in der Bibliothek des Hofzeremonielldepartements enthaltene Archivgut wurde in die Sonderreihe überführt, Kraus (1937) 298. 
Übersichtsplan des Hoftrauerreglements von 1767 mit Beispielen ${ }^{38}$ und des Zeremonielldi rektors von Nepallek: K. 22: Unterlagen zur Stellung des Ersten Obersthofmeisters 1772 und zur Hoftrauer 1792-1881 (Stückverzeichnis in AB XII/7).

Weitere wichtige Bestände des HHStA und anderer Archive

\section{A. HHStA, Hausarchin, Familienakten ${ }^{39}$}

Ein großer Teil der zeremoniellrelevanten Akten wurde zwischen ca. 1878 und 1931 aus dem Bestand der Älteren Zeremonialakten herausgezogen und den Familienakten beigefügt, was man später rückgängig zu machen suchte. ${ }^{40}$ Damit ist freilich angedeutet, daß sich diese Bestände in besonderem Maße überschneiden; die Suche nach einschlägigen zeremoniellrelevanten Quellen lohnt sich in der Regel. Der nach der Revision in den Familienakten verbliebene Bestand an zeremoniellrelevanten Quellen ist immer noch erheblich, die Ordnung erfolgte nach Rubriken, innerhalb derselben chronologisch.

Auswahl von Akten vor 1800, leider meist ohne Angabe der Kartonnummer: Abteilung I. 3. Krönungen 1526-1790, I. 9. Grabstätten und Leichenübertragungen 1491-1822, II. 1. Entbindungen und Taufen 1478-1811, Il. 4. Vermählungen 1422-1823, II. 7. Erkrankungen und Todesfälle 1463-1902, II. 10. Reisen 1449-1840, in K. 96: Bändchen über die Namen der Kinder Maria Theresias, über das Zeremoniell der Entbindungen, Verzeichnis der bei den Kapuzinern begrabenen Habsburger, III. 1. Hofstaatssachen und Zeremoniell 1520-1816, Hofsachen, darin u.a. ein Präzedenzstreit zwischen Erzherzog Leopold Wilhelm und Erzherzog Ferdinand IV. 1645, Hoftrauer 18. Jahrhundert, Hofklagetragungsverordnung 1676, K. 102: ca. 1712 über Hofzeremoniell früherer Zeiten, Zutritt in Antecamera und Ratsstube unter Ferdinand III. und Leopold I., erster Kirchgang der Kaiserin Elisabeth Christine nach der Geburt der Erzherzogin Maria Theresia 1717, Relation einer Konferenz über das Zeremoniell des kaiserlichen Botschafters in Paris 1718, Enfwurf für das Zeremoniell einer Entbindung für Maria Theresia 1741.

Es existiert ein Detailverzeichnis (AB XII/1/2). Siehe auch: HHStA, Familienarchiv, Sammelbände.

B. HHStA, Reichsarchiz, Wahl- und Krönungsakten; Reichsarcbiv, Zeremonialakten; Mainzer Erzanzlerathio im HHSL u a.

Materialien zu Wahlen und Krönungen wurden auch in den Reichsarchiven gesammelt. ${ }^{41}$ Im Bestand Reichsarchiv, Zeremonialakten, finden sich insbesondere Unterlagen zum Zeremoniell im Kontakt mit Reichsfürsten und Gesandten, bei Reichstagen, Bischofswahlen und Thronbelehungen. ${ }^{42}$ Im HHStA befindet sich weiter das besonders für Wahlen und Krönungen wichtige Archiv der Mainzer Erzkanzler., Bei Untersuchungen der Wahl Kaiser Karls VII. und Franz' II. ist auch das Archiv der Grafen von Stadion im HHStA aufschlußreich. ${ }^{44}$ Von Bedeutung für das Gesandtenzeremoniell sind die jeweiligen Länder-

\footnotetext{
39. KRAUS (1937) 282f.

19 Kraus (1937) 21.

4I KRAUS (1937) 296.

4. Ein Inhaltswerzeichn is der umfangreichen Aktensammlung zu Wahl und Krönung von Kaiser Maximilian I. bis Kaiser Franz II. findet sich bei Kratus (1937) 363.

42 Gross (1936) 365f.

43 Vgl. BUCHER (1990) und SCHWERSMANN (1990).

49 REINOHL (1938) 438 .
} 
bestände irn HHStA, für Erbhuldigungen die jeweiligen Teile der Österreichischen bzw. Innerösterreichischen Akten. ${ }^{45}$ Wichtige Quellen liegen auch in den Beständen des Obersthofmarschall-, Obersthofmeister- sowie in weit geringerem Umfang im Archiv des Oberstkämmereramtes. ${ }^{4 k}$

\section{Sonstiges}

Die Bestände des Wiener Hofkammerarchivs zu Fragen des Zeremoniells sind weit weniger ergiebig (vgl. aber die jüngst hervorragend erschlossenen Niederösterreichischen Herrschaftsakten). Dagegen bietet die Österreichische Nationalbibliothek sowohl in threr Sammlung von gedruckten Schilderungen größerer Ereignisse als auch in der Handschriftensammlung reiche Bestände. ${ }^{47}$ Auch die zeremonialwissenschaftlichen Abhandlungen des 18. Jahrhunderts bieten für den Kaiserhof wichtiges Material, das ungeachtet der vielfach unklaren Provenienz der Daten nicht uneingesehen bleiben sollte. ${ }^{48}$ Für Bildmaterial ist die Beizichung der Historischen Blätter der Graphischen Sammlung Albertina (Wien) oft fruchtbar. Hinzuweisen ist zudem auf die oft reichen Materialien in den Familienarchiven der Inhaber hoher und oberster Hofämter, von denen nicht wenige im HHStA und im Allgemeinen Verwaitungsarchiv (Wien) und viele in der Tschechischen Republik liegen. Auch Tagebücher und Kalender von Höflingen können wichtige Quellen zum Hofzeremoniell sein - ediert sind u.a. die aufschlußreichen Tagebücher der Höflinge Khevenhüller-Metsch und Zinzendorf. ${ }^{49}$ Schließlich sei auf die zahlreichen Relationen der auswärtigen Gesandten und Botschafter hingewiesen, die regelmäßig zu Fragen des Zeremoniells Auskunft geben. ${ }^{50}$

\section{Literaturüberblick}

ACADEMIA Caesarea Vindobonensis ( $\mathrm{Hg}$.), Tabulae codicum manuscriptorum praeter graecos et orientales in Bibliotheca Palatina Vindobonensi asservatorum I-VIII. Wien 1864-1893.

Peter J. ARNADE, Realms of ritual: Burgundian ceremony and civic life in late medieval Ghent. Ithaca 1996.

Ronald G. Asch ( $\mathrm{Hg}$.), Der europäische Adel im Ancien Régime. Von der Krise der ständischen Monarchien bis zur Revolution (1600-1789). Köln 2001.

Ronald G. Asch/Adolf M. Birke (Hg.), Princes, patronage, and the nobility. The court at the beginning of the modern age c. 1450-1650. London 1991.

Ronald G. AscH/Heinz DuchHARDT (Hg.), Der Absolutismus - ein Mythos? Strukturwandel monarchischer Herrschaft in West- und Mitteleuropa (ca. 1550-1700). Köln 1996.

Ilsebill BarTa-FliedL/Andreas Gugler/Peter Parenzan (Hg.), Zafeln bei Hof. Zur Geschichte der fürstlichen Tafelkultur. Hamburg 1998.

Beatrix BASTL/Gernot Heiss, Tafeln bei Hof: Die Hochzeitsbankette Kaiser Leopolds I. In: Wiener Geschichtsblätter 50 (1995) 181-206.

Volker BAUER, Die höfische Gesellschaft in Deutschland von der Mitte des 17. bis zum Ausgang des 18. Jahrhunderts. Tübingen 1993.

4 Sowie die entsprechenden Landesarchive.

4. Kraus (1937), Obersthofmeisteramt: 276-295, Oberstkämmereramt: $349-358$, Obersthofmarschallamt:

361-363, Oberststallmeisteramt: 364-367.

47 ACADEMIA (1864-1893) sowie Website der ONB (www.onb.ac.at/sammlungen/hschrift).

48 Zusammenstellungen der wichtigsten Autoren bei VEC (1998) und MüLER (1995) 101-103. Für den Kaiser-

hof von besonderer Bedeutung sind Stieve, vgl. VEC (1998) 43-49, sowie KUCHELBECKER (1730).

49 KhevenHUUlLer-Metsch (1907-1972); Breunlich/Mader (1997).

so Vgl. zur Erschließung BITNER/GRoss (1936). 
Volker BAuEr, Hofökonomie. Der Diskurs über den Fürstenhof in Zeremonialwissenschaft, Hausväterliteratur und Kameralismus. Wien-Köln-Weimar 1997.

Andréa BeILIGER/David J. Krieger (Hg.), Ritualtheorien. Opladen-Wiesbaden 1998.

Christian BENEDIK, Die herrschaftlichen Appartements. Funktion und Lage während der Regierungen von Kaiser Leopold I. bis Kaiser Franz Joseph I. In: Österreichische Zs. für Kunst und Denkmalpflege 51 (1997) 552-570.

Christian BENEDIK, Die Repräsentationsräume der Wiener Hofburg in der ersten Hälfte des 18. Jahrhunderts. In: Das siebzehnte Jahrhundert in Österreich 2(1997) 7-22=BENEDIK (1997a).

Jörg Jochen BERNS/Thomas RAHN (Hg.), Zeremoniell als höfische Ästhetik in Spätmittelalter und früher Neuzeit. Tübingen 1995.

Ludwig BrTtner/Lothar Gross $(\mathrm{Hg}$.), Repertorium der diplomatischen Vertreter aller Länder seit dem Westfalischen Frieden, 2 Bde. Berlin 1936.

Georg BRAUNGART, Hofberedsamkeit. Studien zur Praxis höfisch-politischer Rede im deutschen Territorialabsolutismus. Tübingen 1988.

Maria BreunLICH/Marieluise MADER ( $\mathrm{Hg}$.), Karl von Zinzendorf. Aus den Jungendtagebüchern. 1747, 1752 bis 1763 . Wien 1997.

Michael BRIX, Trauergerüste für die Habsburger in Wien. In: Wiener Jb. für Kunstgeschichte 26 (1973) 201-265.

Editha BUCHER ( $\mathrm{Hg}$.), Inventar des Mainzer Reichserzkanzler-Archivs im Haus-, Hof- und Staatsarchiv Wien aufgrund des Verzeichnisses von Wilhelm Klemm. Mit einer Einleitung zur Geschichte des Archivs von Leopold Auer. Koblenz 1990.

August Buck/Georg KauFrmann/Blake Lee SpAHR/Conrad WIEDEMANn (Hg.), Europäische Hofkultur im 16. und 17. Jahrhundert. Vorträge und Referate gehalten anläßlich des Kongresses des Wolfenbütteler Arbeitskreises für Renaissanceforschung und des Internationalen Arbeitskreises für Barockliteratur, 3 Bde. Hamburg 1981.

Monique ChATENET, La Cour de France au XVI siècle. Vie sociale et architecture. Paris 2002. Jeroen DuindaM, Myths of power. Norbert Elias and the early modern european court. Amsterdam 1994.

Jeroen DuINDAM, Norbert Elias und der frühneuzeitliche Hof. Versuch einer Kritik und Weiterführung. In: HA 6 (1998) 370-387.

Jeroen DuINDAM, The Bourbon and Austrian Habsburg Courts. Numbers, Ordinances, Ceremony and Nobles. In: AsCH (2001) 181-206.

Jeroen DUINDAM, Ceremonial staffs and paperwork at two courts: France and the Habsburg monarchy ca. 1550-1720. In: MALETTKE/GRELI (2001) 369-388 = DUINDAM (2001a).

Hubert Ch. EHAlT, Ritus und Rationalität im Herrschaftsstil des 17, und 18. Jahrhunderts. In: Beiträge zur historischen Sozialkunde 8 (1977) 8-11.

Hubert Ch. EHAlT, Ausdruckformen absolutischer Herrschaft. Der Wiener Hof im 17. und 18. Jahrhundert. Wien-München 1980.

Norbert ELIAS, Die höfische Gesellschaft. Untersuchungen zur Soziologie des Königtums und der höfischen Aristokratie. Mit einer Einleitung: Soziologie und Geschichtswissenschaft. Frankfurt am Main 1983.

Erika FisCHER-LiCHTE, Ästhetische Erfahrung. Das Semiotische und das Performative. Tübingen-Basel 2001.

Birgit FrANKE, Alttestamentliche Tapisserien und Zeremoniell am burgundischen Hof. In: BERNS/RAHN (1995) 332-352. 
Gotthardt FRÜHSORGE, Der Hof, der Raum, die Bewegung. Gedanken zur Neubewertung des europäischen Hofzeremoniells. In: Euphorion. Zs. für Literaturgeschichte 82 (1988) 424-265.

Ursula GErrNer, Die Sprache der Verstellung. Studien zum thetorischen und anthropologischen Wissen im 17. und 18. Jahrhundert. Tübingen 1992.

Maria Goloubeva, The glorification of emperor Leopold I in image, spectacle and text. Mainz 2000.

Henriette GraF, Das kaiserliche Zeremoniell und das Repräsentationsappartement im Leopoldinischen Trakt der Wiener Hofburg um 1740. In: Österreichische Zs. für Kunst und Denkmalpflege 51 (1997) 571-587.

Lothar Groß, Die Reichsarchive. In: Ludwig Bittner (Hg), Inventare des Wiener Haus-, Hofund Staatsarchivs. Gesamtinventar des Wiener Haus-, Hof- und Staatsarchivs, Bd. 1. Wien 1936, 273-394.

Ingrid HASUINGER, Der Kaiser speist en public. Die Geschichte der offentlichen Tafel bei den Habsburgern vom 16. bis ins 20. Jahrhundert. In: Hans Ottomeyer/Michaela Völkel ( $\mathrm{Hg}$ ), Die öffentliche Tafel. Tafelzeremoniell in Europa 1300-1900. Wolfrathshausen 2002, 48-57.

Christian HattenHauer, Wahl und Krönung Franz II. AD 1792. Das Heilige Römische Reich krönt seinen letzten Kaiser - Das Tagebuch des Reichsquartiermeisters Hieronymus Gottfried von Müller und Anlagen. Frankfurt am Main-Berlin-Bern-New York 1995.

Magdalena HAWLIK-VAN DE WATER, Der schöne Tod. Zeremonialstrukturen des Wiener Hofes bei Tod und Begräbnis zwischen 1640 und 1740. Wien-Freiburg-Basel 1989.

Mark HENGERER, Hofzeremoniell, Organisation und Grundmuster sozialer Differenzierung am Wiener Hof im 17, Jahrhundert. In: MALETTKE/GreLL (2001) 337-368.

Mark Hengerer, Kaiserhof und Adel in der Mitte des 17. Jahrhunderts. Eine Kommunikationsgeschichte der Macht in der Vormoderne. Diss. Konstanz 2002.

Nicholas HENSHAL, The myth of absolutism. Change and continuity in early modern european monarchy. London-New York 1992.

Jan HIRSCHBIEGEI, Auswahlbibliographie von Neuerscheinungen zu Residenz und Hof 1995-2000. Kiel 2000.

Christina HofMANN, Das spanische Hofzeremoniell von 1500-1700. Frankfurt am MainBern-New York 1985.

Christina HoFMANN, Das Spanische Hofzeremoniell - eine spezifische Ausdrucksform nichtverbaler Sprache. In: Volker Kapp ( $\mathrm{Hg}$.), Die Sprache der Zeichen und Bilder: Rhetorik und nonverbale Kommunikation in der frühen Neuzeit. Marburg 1990, 142-148.

Christina Hofmann-Randal, Die Herkunft und Tradierung des Burgundischen Hofzeremoniells. In: BERNS/RAHN (1995) 150-156.

André HoLEnsteIn, Die Huldigung der Untertanen. Rechtskultur und Herrschaftsordnung (800-1800). Stuttgart-New York 1991.

André HOLENSTEIN, Huldigung und Herrschaftszeremoniell im Zeitalter des Absolutismus und der Aufklärung. In: Aufklärung 6/2 (1991) 21-46 = HOLENSTEIN (1991a).

Bernhard JAHN/Thomas RaHN/Claudia SCHNTTZER (Hg.), Zeremoniell in der Krise. Störung und Nostalgie. Marburg 1998.

Rudolf Graf KHEVENHÜLER-Metsch/Hanns SCHLTter/Maria BREunilch-PAWLIK (Hg.), Aus der Zeit Maria Theresias. Tagebuch des Fürsten Johann Josef Khevenhüller-Metsch, 8 Bde. Wien-Leipzig 1907-1972. 
Helmuth KIESEL, „Bei Hof, bei Höll.“ Untersuchungen zur literarischen Hofkritik von Sebastian Brant bis Friedrich Schiller. Tübingen 1979.

André KIESERLING, Kommunikation unter Anwesenden. Studien über Interaktionssysteme. Frankfurt am Main 1999.

Samuel John KLINGENSMTrH, The utility of splendor. Ceremony, social life, and architecture at the court of Bavaria, 1600-1800. Chicago-London 1993.

Sabine KOLOCH, Zeremoniellbücher als Forschungsaufgabe kulturhistorischer Frauenforschung. In: kritische berichte. Zs. für Kunst- und Kulturwissenschaften 24/4 (1996) 43-60.

Elisabeth KovÁCs, Kirchliches Zeremoniell am Wiener Hof des 18. Jahrhunderts im Wandel von Mentalität und Gesellschaft. In: MÖStA 32 (1979) 109-142.

Wilhelm Kraus, Die Hofarchive. In: Ludwig Bittner ( $\mathrm{Hg}$.), Inventare des Wiener Haus-, Hofund Staatsarchivs. Gesamtinventar des Wiener Haus-, Hof- und Staatsarchivs, Bd. 2. Wien 1937, 273-375.

Jürgen KrUEDENER, Die Rolle des Hofes im Absolutismus. Stuttgart 1973.

Johann Basilius KUCHELBECKER, Allerneueste Nachricht vom Römisch=Kayserl. Hofe nebst einer ausführlichen Historischen Beschreibung der kayserlichen Residentz=Stadt Wien und der umliegenden Oerter [...]. Hannover 1730.

Joachim LEHNEN, Adventus principis. Untersuchungen zu Sinngehalt und Zeremoniell der Kaiserankunft in den Städten des Imperium Romanum. Frankfurt am Main u. a. 1997.

Uta LOWENSTEIN, Vorraussetzungen und Grundlagen von Tafelzeremoniell und Zeremonientafel. In: BERNS/RAHN (1995) 266-279.

Niklas LuHmanN, Gesellschaftsstruktur und Semantik. Studien zur Wissenssoziologie der modernen Gesellschaft I. Frankfurt am Main 1993.

Klaus MALETTKE/Chantal GREL (Hg.), Hofgesellschaft und Höflinge an europäischen Fürstenhöfen in der frühen Neuzeit (15.-18. Jahrhundert). Münster-Hamburg-Berlin-London 2001.

Aloys MEISTER, Kleiner Beitrag zur Geschichte der Nuntiaturen. Ceremoniell der Nuntien. In: Römische Quartalschrift für christliche Altertumskunde und Kirchengeschichte 5/ Nr. 2 (1891) 159-178.

Ferdinand MENČ́k, Beiträge zur Geschichte der kaiserlichen Hofämter. In: AÖG 87 (1899) 447-564.

Oskar Freiherr von Mrns, Gundacker von Liechtensteins Anteil an der kaiserlichen Zentralverwaltung (1606-1654). In: Beiträge zur neueren Geschichte Österreichs 4 (Wien 1908) $35-118$.

Rainer A. MÜLER, Der Fürstenhof in der frühen Neuzeit. München 1995.

Werner Paravicin, The court of the dukes of Burgundy: a model for Europe: In: AsCH/ BIRKE (1991) 69-102.

Werner Paravicini (Hg.), Zeremoniell und Raum. 4. Symposium der Residenzen-Kommission der Akademie der Wissenschaften in Göttingen. Sigmaringen 1997.

Agostino Paravicini Bagliani, Der Leib des Papstes. Eine Theologie der Hinfälligkeit. München 1997.

Karin PLODECK, Hofstruktur und Hofzeremoniell in Brandenburg-Ansbach vom 16. bis zum 18. Jahrhundert. Zur Rolle des Herrschaftskultes im absolutistischen Gesellschaftsund Herrschaftssystem. Ansbach 1972.

Friedrich B. Pousross, Des abwesenden Prinzen Porträt. Zeremonielldarstellung im Bildnis und Bildnisgebrauch im Zeremoniell. In: BERNS/RAHN (1995) 382-409. 
Friedrich B. POLEROSS, Tradition und Recreation. Die Residenzen der österreichischen Habsburger in der frühen Neuzeit (1490-1780). In: Majestas 6 (1998) 91-148.

Kurt PüCHL, Die Erbhuldigungen der niederösterreichischen Stände im 17., 18. und 19. Jahrhundert in Wien. Diss. Wien 1954.

Thomas RaHN, Herrschaft der Zeichen. Zum Zeremoniell als "Zeichensystem“. In: Hans Ottomeyer/Michaela Volkel (Hg.), Die offentliche Tafel. Tafelzeremoniell in Europa 1300-1900. Wolfrathshausen 2002, 22-31.

Fritz von ReINÖH, Die unter Vorbehalt des Privateigentumsrechtes hinterlegten Archivkörper und Sammlungen. In: Ludwig Bittner $(\mathrm{Hg}$.$) , Inventare des Wiener Haus-, Hof- und$ Staatsarchivs. Gesamtinventar des Wiener Haus-, Hof- und Staatsarchivs, Bd. 4. Wien $1938,407-448$.

Helga REUTER-PETIENBERG, Bedeutungswandel der römischen Königskrönung in der Neuzeit. Diss. Köln 1963.

Bernd RIL, KarlVI. Habsburg als barocke Großmacht. Graz-Wien-Köln 1992.

Gerrit Jasper SCHENK, Zeremoniell und Politik. Herrschereinzüge im spätmittelalterlichen Reich. Köln-Weimar-Wien 2003.

Henry Frederick SCHWARZ, The imperial privy council in the seventeenth century. Cambridge Mass. 1943.

Gerd SCHWERHOFF, Zivilisationsprozeß und Geschichtswissenschaft. Norbert Elias' Forschungsparadigma in historischer Sicht. In: HZ 266 (1998) 561-605.

Aloys SCHWERSMANN (Hg.), Inventar des Mainzer Regierungs-Archivs, Bd. 1: Kurfürstentum und Reich. Zentralbehörden. Koblenz 1990.

Herbert SEIFERT, Die Oper am Wiener Kaiserhof im 17. Jahrhundert. Tutzing 1985.

Stefan SIENEu, Die Geheime Konferenz unter Kaiser Leopold I. Personelle Strukturen und Methoden zur politischen Entscheidungsfindung am Wiener Hof. Frankfurt/Main u.a. 2001.

Andrea SOMMER-MATHIS, Theatrum und Ceremoniale. Rang-und Sitzungsordnungen bei theatralischen Veranstaltungen am Wiener Kaiserhof im 17. und 18. Jahrhundert. In: BERNS/ RAHN (1995) 511-533.

Justin STAGL, Ritual, Zeremoniell, Etikette. In: Jb. für Volkskunde 13 (1990) 7-21.

Waltraud STANGL, Tod und Trauer bei den österreichischen Habsburgern 1740-1780 dargestellt im Spiegel des Hofzeremoniells. Diss. Wien 2001.

Angela STǑCKELE, Taufzeremoniell und politische Patenschaften am Kaisethof. In: MIÖG 90 (1982) 271-337.

Barbara STOLBERG-RILINGER, Höfische Öffentlichkeit. Zur zeremoniellen Selbstdarstellung des brandenburgischen Hofes vor dem europäischen Publikum. In: Forschungen zur brandenburgischen und preussischen Geschichte NF 7/Nr. 2 (1997) 145-176.

Barbara Stollberg-Riunger (Hg.), Vormoderne politische Verfahren. Berlin 2001.

Michael STÜRMER, Gehäuse der höfischen Gesellschaft. In: ZHF 7 (1980) 219-228.

Miloš VEC, Zeremonialwissenschaft im Fürstenstaat. Studien zur juristischen und politischen Theorie absolutistischer Herrschaftsrepräsentation. Frankfurt am Main 1998.

Karl VOCELKA, Habsburgische Hochzeiten 1550-1600. Kulturgeschichtliche Studien zum manieristischen Repräsentationsfest. Köln-Wien-Graz 1976.

Karl VoCELKA, Glanz und Untergang der höfischen Welt. Repräsentation, Reform und Reaktion im habsburgischen Vielvölkerstaat. Wien 2001.

Karl VocelKa/Lynne HeLer, Die private Welt der Habsburger. Leben und Alltag einer Familie. Graz-Wien-Köln 1998. 
Bernd Herbert WANGER, Kaiserwahl und Krönung im Frankfurt des 17. Jahrhunderts. Darstellung anhand der zeitgenössischen Bild- und Schriftquellen und unter besonderer Berücksichtigung der Erhebung des Jahres 1612. Frankfurt am Main 1994.

Martin WARNKE, Hofkünstler. Zur Vorgeschichte des modernen Künstlers. Köln ${ }^{2} 1996$.

Thomas WINKelbauER, Fürst und Fürstendiener. Gundaker von Liechtenstein, ein österreichischer Aristokrat des konfessionellen Zeitalters. Wien-München 1999.

Aloys WINTERLING, Der Hof der Kurfürsten von Köln 1688-1794. Eine Fallstudie zur Bedeutung „absolutistischer“ Hofhaltung. Bonn 1986.

Aloys WINTERUNG, Die frühneuzeitlichen Höfe in Deutschland. Zur Lage der Forschung. In: Internationales Archiv für Sozialgeschichte der deutschen Literatur 21 (1996) 181-189.

Uwe WIRTH ( $\mathrm{Hg}$.), Performanz. Zwischen Sprachphilosophie und Kulturwissenschaften. Frankfurt am Main 2002.

Dmitri ZACHAR'N, Symbolische Körperhaltungen. Eine Differenz zwischen russischen und westeuropäischen Zeremonial-,Grammatiken" des 16. und 17. Jahrhunderts. In: Margreth Egidi/Oliver Schneider/Matthias Schöning/Irene Schütze/Caroline Torra-Mattenklott (Hg.), Gestik. Figuren des Körpers in Text und Bild. Tübingen 2000, 87-102.

Ivan von ŽOLGER, Der Hofstaat des Hauses Österreich. Wien-Leipzig 1917. 